\title{
Brain-derived neurotrophic factor-TrkB signaling in the medial prefrontal cortex plays a role in the anhedonia-like phenotype after spared nerve injury
}

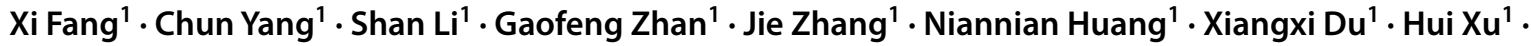 \\ Kenji Hashimoto ${ }^{2}$. Ailin Luo ${ }^{1}$
}

Received: 11 April 2018 / Accepted: 31 May 2018 / Published online: 7 June 2018

(c) The Author(s) 2018

\begin{abstract}
Although depressive symptoms including anhedonia (i.e., loss of pleasure) frequently accompany pain, little is known about the risk factors contributing to individual differences in pain-induced anhedonia. In this study, we examined if signaling of brain-derived neurotrophic factor (BDNF) and its receptor tropomyosin-receptor-kinase B (TrkB) contribute to individual differences in the development of neuropathic pain-induced anhedonia. Rats were randomly subjected to spared nerved ligation (SNI) or sham surgery. The SNI rats were divided into two groups based on the results of a sucrose preference test. Rats with anhedonia-like phenotype displayed lower tissue levels of BDNF in the medial prefrontal cortex (mPFC) compared with rats without anhedonia-like phenotype and sham-operated rats. In contrast, tissue levels of BDNF in the nucleus accumbens (NAc) of rats with an anhedonia-like phenotype were higher compared with those of rats without anhedonia-like phenotype and sham-operated rats. Furthermore, tissue levels of BDNF in the hippocampus, L2-5 spinal cord, muscle, and liver from both rats with or without anhedonia-like phenotype were lower compared with those of sham-operated rats. A single injection of 7,8-dihydroxyflavone (10 mg/kg; TrkB agonist), but not ANA-12 (0.5 mg/kg; TrkB antagonist), ameliorated reduced sucrose preference and reduced BDNF-TrkB signaling in the $\mathrm{MPFC}$ in the rats with anhedonia-like phenotype. These findings suggest that reduced BDNF-TrkB signaling in the mPFC might contribute to neuropathic pain-induced anhedonia, and that TrkB agonists could be potential therapeutic drugs for pain-induced anhedonia.
\end{abstract}

Keywords Anhedonia $\cdot$ BDNF $\cdot$ Individual differences $\cdot$ Neuropathic pain $\cdot \operatorname{TrkB}$

\section{Introduction}

Pain is one of the most common ailments that make patients seek medical treatment, representing a major clinical, social, and economic problem. Pain and depression frequently

Xi Fang and Chun Yang have contributed equally to this study.

Kenji Hashimoto

hashimoto@faculty.chiba-u.jp

$\triangle$ Ailin Luo

alluo@tjh.tjmu.edu.cn

1 Department of Anesthesiology, Tongji Hospital, Tongji Medical College, Huazhong University of Science and Technology, No. 1095 Jiefang Avenue, Wuhan 430030, China

2 Division of Clinical Neuroscience, Chiba University Center for Forensic Mental Health, Chiba 260-8670, Japan manifest together. Epidemiological studies have shown that the prevalence of depression in patients with pain is higher compared with that when these two conditions are separately evaluated [1-3]. Approximately $30 \%$ of patients in pain experience depression $[4,5]$. A recent longitudinal analysis demonstrated that chronic pain strongly predicts the development of more depressive symptoms in patients with pain compared with those without pain [6]. These epidemiological studies suggest that individual differences exist in the development of pain and depression comorbidities [1, 2, 7-9]. Although pain and depression share biological pathways, the precise mechanisms underlying the comorbidity of pain and depression remain unknown. In addition, the possible predisposing factors for individual differences in these comorbidities remain poorly understood.

The signaling pathway comprising brain-derived neurotrophic factor (BDNF) and its specific receptor tropomyosin-receptor-kinase B (TrkB) plays a key role in the 
pathophysiology of depression and in the antidepressant functions of antidepressants [10-18]. Alterations in BDNF-TrkB signaling in the brain have been implicated in the pathogenesis of depression and antidepressant mechanisms. Decreased BDNF-TrkB signaling in the prefrontal cortex (PFC) and hippocampus was shown in conditions of chronic unpredictable mild stress (CUMS) [19], inflammation [20-22], chronic social defeat stress (CSDS) [23-25], and learned helplessness (LH) [26, 27]. In contrast, increased BDNF-TrkB signaling in the nucleus accumbens (NAc) was detected in inflammation [20,21], CSDS [23-25], and LH models [26, 27]. Recent studies suggest that regional differences in BDNF-TrkB signaling confer resilience to inescapable stress [27, 28]. Accumulating evidence suggests that BDNF-TrkB signaling also plays a crucial role in pain $[29,30]$. A recent study showed that alterations in inflammatory cytokines and BDNF might contribute to neuropathic pain-induced depression [31]. However, little is known about the detailed role of BDNF-TrkB signaling in individual emotional responses to peripheral nerve injury.

According to the Diagnostic and Statistical Manual of Mental Disorders (DSM-5), the two core symptoms of depression are depressed mood and anhedonia. It is important to investigate the role of anhedonia in pain, because it specifically predicts worse treatment outcome [32] and a longer and more severe course of depression [33]. The purpose of the current study was to examine whether BDNFTrkB signaling might contribute to the individual differences in anhedonia-like phenotype in rats after spared nerve injury (SNI). Furthermore, we examined if 7,8-dihydroxyflavone (7,8-DHF; a TrkB agonist) [20, 26, 34] or ANA-12 (a TrkB antagonist) [20, 26, 35, 36] could improve anhedonia-like symptoms and alterations in BDNF-TrkB signaling in anhedonia-susceptible rats after SNI.

\section{Materials and methods}

\section{Animals}

Male Sprague Dawley (SD) rats (weighing 180-230 g) were purchased from the Laboratory Animal Centre of Tongji Medical College, Huazhong University of Science and Technology (Wuhan, China). The animals were housed under $12 \mathrm{~h} \mathrm{light/dark} \mathrm{cycle} \mathrm{with} \mathrm{free} \mathrm{access} \mathrm{to} \mathrm{food} \mathrm{and} \mathrm{water.} \mathrm{Pro-}$ cedures of this animal experiment were in accordance with the National Institute of Health Guide for the Care and Use of Laboratory Animals. The experimental protocols were approved by the Experimental Animal Committee of Tongji Hospital, Tongji Medical College, Huazhong University of Science and Technology.

\section{Experimental design}

As shown in Fig. 1a, rats were acclimated to environment for 6 days. Then, the mechanical withdrawal threshold (MWT) was performed 1 day before the spared nerve injury (SNI) surgery for baseline measurement. MWT was implemented from days 6,12 , and 19 after surgery. Sucrose preference test (SPT) was implemented from days 7, 14, and 21 after surgery. 23 days after SNI surgery, medial prefrontal cortex (mPFC), hippocampus and nucleus accumbens (NAc) of brain, liver, muscle, and L2-5 spinal cord tissues were collected. In addition, in Fig. 5a, anhedonia-susceptible rats were intraperitoneally injected with either 7,8-dihydroxyflavone (7,8-DHF, a TrkB agonist) or ANA-12, (N2-(2-\{[(2-oxoazepan-3-yl) amino]carbonyl \} phenyl)benzo[b]thiophene-2-carboxamide, a TrkB antagonist). 7,8-DHF (Catalog number: ab120996, Abcam, UK, $10 \mathrm{mg} / \mathrm{kg}$ ), and ANA-12 (Catalog number: HY-12497, MedChem Express, USA, $0.5 \mathrm{mg} / \mathrm{kg}$ ) were prepared in vehicle of $17 \%$ dimethyl sulfoxide (DMSO) in phosphatebuffered saline. The SPT and MWT were performed on days 12 and 14, respectively. On day 15, mPFC and NAc of rats were collected for measurement.

\section{SNI}

The SNI surgery was performed as previously described [37]. Rats were anesthetized with $10 \%$ chloral hydrate $(3 \mathrm{ml} / \mathrm{kg}$ ) and then the skin of left thigh was incised. The sciatic nerve and its three terminal branches after bluntly dissecting biceps femoris muscle were totally exposed. The common peroneal and tibial nerves were ligated with a 4-0 silk and cut off the distal to the ligation. The muscle and skin were sutured with a 4-0 silk. Rats in the sham group were exposed to the sciatic nerve and its three terminal branches but without ligated, and cut off the common peroneal and tibial nerves.

\section{MWT}

Before MWT, rats were placed in plexiglass chambers with a wire net floor for 30 min avoiding the stress resulting from the test conditions. The Electronic Von Frey (UGO BASILE S.R.L., Italy) filaments were applied to the lateral $1 / 3$ of right paws. The paws' quick withdrawal or flinching was considered as a positive response. Every filament stimuli were applied four times with a period of $30 \mathrm{~s}$ interval. 
A

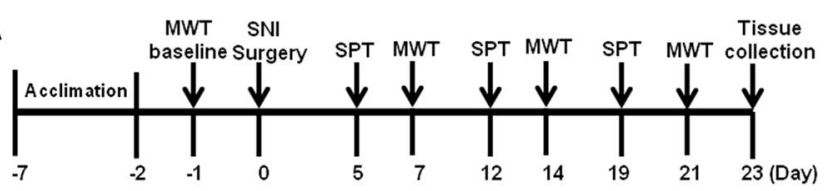

B

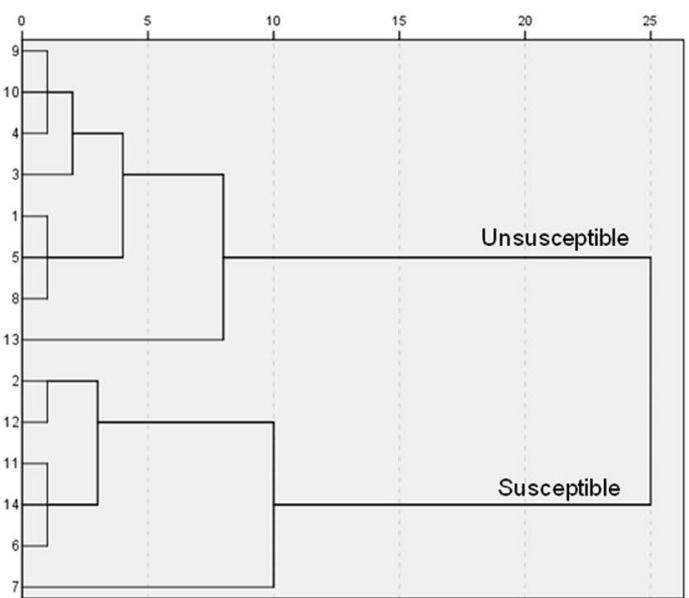

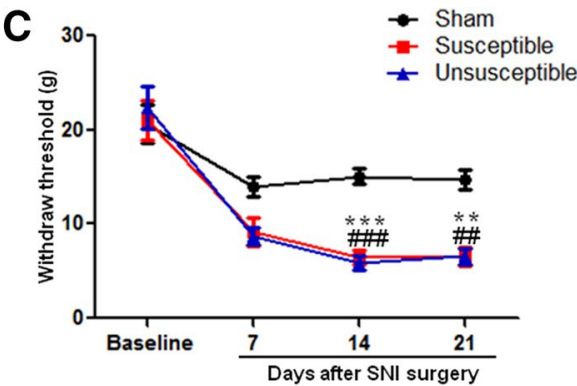

D

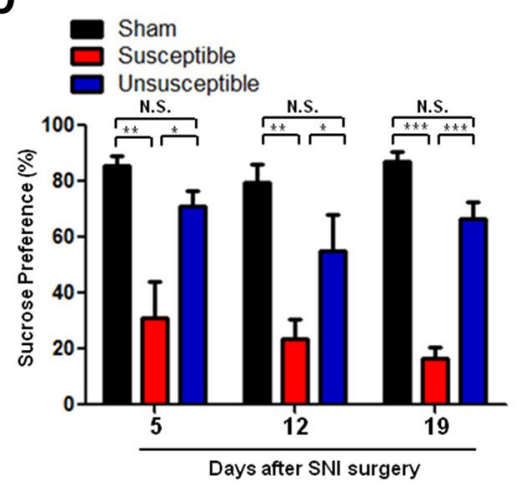

Fig. 1 Comparisons of MWT and SPT in sham, anhedonia susceptible, and unsusceptible rats. a Schedule of SNI, MWT, and SPT. SNI was performed on day 0 after 7 day acclimation. MWT was measured on days 7, 14, and 21 after SNI, respectively. SPT was performed on days 5, 12, and 19 after SNI, respectively. b Dendrogram of hierarchical clustering analysis. A total of 14 SNI rats were divided into anhedonia susceptible and unsusceptible groups by SPT results of hierarchical clustering analysis. c MWT (time: $F_{(3,15)}=93.989, P<0.001$; Group: $\quad F_{(2,10)}=14.526, \quad P=0.001$; interaction: $F_{(6,30)}=3.974$, $P=0.005$ ) was measured on days 7,14 , and 21 in the sham, anhedonia unsusceptible, and anhedonia susceptible groups after SNI,

\section{SPT}

Rats were exposed to water and $1 \%$ sucrose solution for $48 \mathrm{~h}$, followed by $24 \mathrm{~h}$ of water and food deprivation and a $24 \mathrm{~h}$ exposure to two identical bottles, one is water, and another is $1 \%$ sucrose solution. The bottles containing water and sucrose were weighed before and at the end of this period and the sucrose preference was determined.

\section{Western blot}

Samples were homogenized with RIPA buffer $(150 \mathrm{mM}$ sodium chloride, Triton X-100, $0.5 \%$ sodium deoxycholate, $0.1 \%$ sodium dodecyl sulfate, $50 \mathrm{mM}$ Tris, and $\mathrm{pH}$ 8.0 ) at $4{ }^{\circ} \mathrm{C}$ for $30 \mathrm{~min}$, and were then centrifuged for 15 min at $4{ }^{\circ} \mathrm{C}$. BCA protein assay kit (Boster, Wuhan, China) was used to determine the protein levels in supernatant. The samples were separated by $10 \%$ sodium dodecyl sulfate-polyacrylamide gel electrophoresis and respectively. Data are shown as mean \pm S.E.M. $(n=6-8) . * * P<0.01$ or $* * * P<0.001$, susceptible group vs sham group. ${ }^{\# \#} P<0.01$ or ${ }^{\# \# \#} P<0.001$, unsusceptible group vs sham group. d SPT (time: $F_{(2,10)}=2.723, P=0.114$; Group: $F_{(2,10)}=51.176, P<0.001$; interaction: $\left.F_{(4,20)}=0.424, P=0.790\right)$ was measured in the sham, anhedonia unsusceptible, and anhedonia susceptible groups on days 5, 12, and 19 after SNI, respectively. Data are shown as mean \pm S.E.M. $(n=6-8)$. $* * * P<0.05, * * P<0.01$ or $* * * P<0.001$. MWT mechanical withdrawal test, N.S. not significant, $S N I$ spared nerve injury, $S P T$ sucrose preference test

were transferred to polyvinylidene fluoride membranes (Millipore, Bedford, MA, USA). Bands were blocked with $5 \%$ BSA in TBST $(0.1 \%$ Tween 20 in Tris-buffered saline) for $1 \mathrm{~h}$ at room temperature. Relative primary antibodies were incubated at $4{ }^{\circ} \mathrm{C}$ overnight: rabbit mature BDNF (1:500, Sangon Biotech, Shanghai, China), rabbit proBDNF (1:500; Origene, Rockville, MD, USA), rabbit TrkB (1:1000, Cell Signaling Technology, Danvers, MA, USA), rabbit phosphorylated p-TrkB (1:1000, Affinity, Cincinnati, OH, USA), and mouse GAPDH (1:1000, Qidongzi, Wuhan, China). Then, bands were washed with TBST and incubated second antibody for $2 \mathrm{~h}$ at room temperature: goat anti-rabbit IgG horseradish peroxidase or goat anti-rabbit $\operatorname{IgG}$ horseradish peroxidase (1:5000, Qidongzi, Wuhan, China). Finally, these bands were detected by enhanced chemiluminescence reagents (Qidongzi, Wuhan, China) with the ChemiDocXRS chemiluminescence imaging system (Bio-Rad, Hercules, CA, USA). 


\section{Statistical analyses}

The data show as the mean \pm standard error of the mean (SEM). Analysis was performed using PASW Statistics 20 (formerly SPSS Statistics; SPSS). Comparisons between groups were performed using the one-way analysis of variance (ANOVA) or two-way ANOVA, followed by post hoc Tukey test. Hierarchical cluster analysis of SPT was applied to classify the anhedonia susceptible or unsusceptible rats. The $P$ values of less than 0.05 were considered statistically significant.

\section{Results}

\section{The results of WMT and SPT among the sham, anhedonia susceptible, and unsusceptible rats}

Anhedonia susceptible and unsusceptible rats were divided by hierarchical cluster analysis of sucrose preference test (Fig. 1b). WMT was significantly decreased in both anhedonia susceptible and unsusceptible rats as compared with that of sham on days 7, 14, and 21 after SNI surgery (Fig. 1c). However, there was no any change in the WMT between anhedonia susceptible and unsusceptible rats, although WMT in the both groups was significantly lower than sham group (Fig. 1c). Furthermore, anhedonia susceptible rats showed a significant decrease in SPT as compared with those in the sham and unsusceptible rats on days 5, 12, and 19 after SNI, respectively (Fig. 1d).

\section{Alterations in the tissue levels of BDNF in selected tissues}

As shown in Fig. 2, there were alterations in the BDNF levels in mPFC, NAc, hippocampus, L2-5 spinal cord, muscle, and liver in the rats with SNI-induced anhedonia. Post hoc analysis showed that anhedonia susceptible rats significantly lower levels of mature BDNF in the mPFC than those in sham or anhedonia unsusceptible groups (Fig. 2a). In contrast, anhedonia susceptible rats demonstrated significant higher levels of mature BDNF in the NAc than those in sham or unsusceptible groups. Furthermore, tissue levels of mature BDNF in the L2-5 spinal cord, muscle, and liver of
A
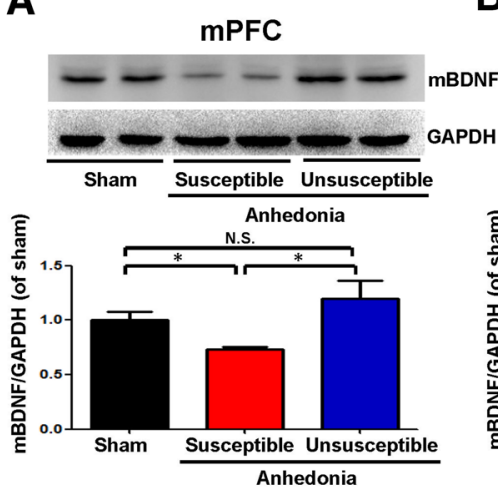

D

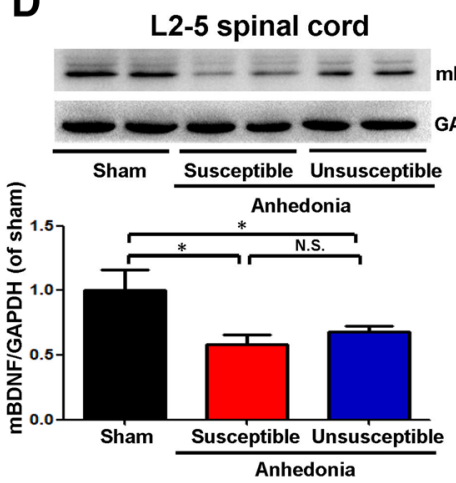

B
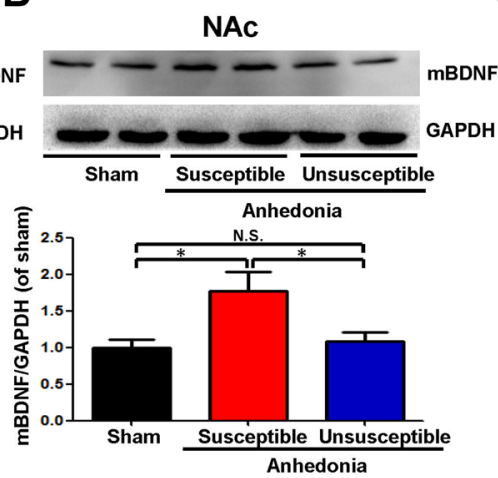

E

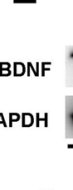

C

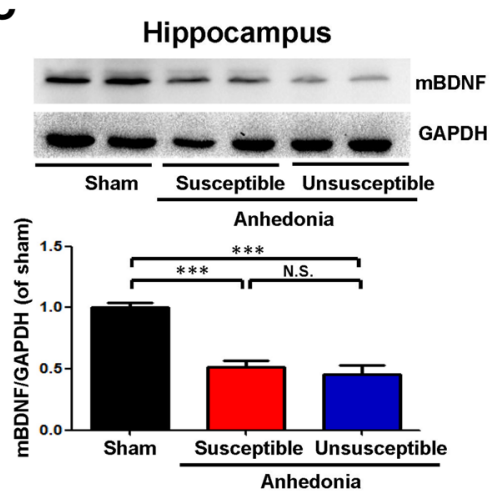

$\mathbf{F}$
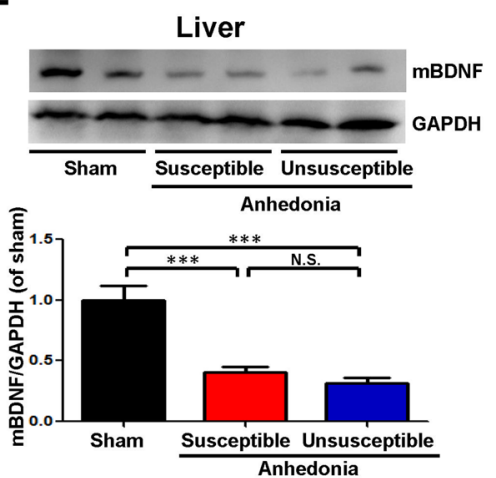

Fig. 2 Levels of BDNF in selected tissues among the sham, anhedonia susceptible and unsusceptible groups. a mPFC $\left(F_{(2,15)}=6.108\right.$, $P=0.011)$. b NAc $\left(F_{(2,15)}=5.8, \quad P=0.014\right)$. c Hippocampus $\left(F_{(2,15)}=59.856, \quad P<0.001\right)$; d L2-5 spinal cord $\left(F_{(2,15)}=4.307\right.$, $P=0.033)$. e Muscle $\left(F_{(2,15)}=8.166, \quad P=0.004\right)$. f Liver
$\left(F_{(2,15)}=28.928, P<0.001\right)$. Data are shown as mean \pm S.E.M. $(n=6)$. $* P<0.05, * * P<0.01$ or $* * * P<0.01$. BDNF brain-derived neurotrophic factor, $m P F C$ medial prefrontal cortex, NAc nucleus accumbens, N.S. not significant 
anhedonia susceptible and unsusceptible rats were significantly lower than those on sham group (Fig. 2d-f).

\section{Alterations in the tissue levels of proBDNF in selected tissues}

Mature BDNF is prepared from its precursor proBDNF. Tissue levels of proBDNF were significantly lower in the mPFC and L2-5 spinal cord of anhedonia susceptible rats compared sham or unsusceptible groups (Fig. 3a, d). In contrast, tissue levels of proBDNF in the NAc from anhedonia susceptible rats were significantly higher than those of sham or unsusceptible rats. Tissue levels of proBDNF in the hippocampus and liver from anhedonia susceptible rats were significantly lower than those of sham rats, although there was no change between anhedonia susceptible rats and unsusceptible rats (Fig. 3c, f). Furthermore, there was no difference in proBDNF in the muscle among three groups (Fig. 3e)

\section{Alterations in phosphorylated p-TrkB and TrkB levels in the selected tissues}

To clarify whether TrkB activation or inhibition underpins anhedonia-like behavior in SNI rats, we performed immunoblot analyses of TrkB and phosphorylated TrkB ( $\mathrm{p}$-TrkB), an activated form of TrkB, in samples from the mPFC, NAc, hippocampus, L2-5 spinal cord, muscle, and liver. The anhedonia susceptible rats, but not unsusceptible rats, had lower p-TrkB/TrkB ratio in the mPFC (Fig. 4a). In addition, both anhedonia susceptible and unsusceptible rats had lower $\mathrm{p}-\mathrm{TrkB} / \mathrm{TrkB}$ ratio in the hippocampus, L2-5 spinal cord, muscle, and liver (Fig. 4c-f). In contrast, $\mathrm{p}-$ TrkB/TrkB ratio in the NAc in the anhedonia susceptible rats was significantly higher than those of sham rats or anhedonia unsusceptible rats (Fig. 4b). However, there were no significant changes of total TrkB levels in the all regions among the three groups.
A

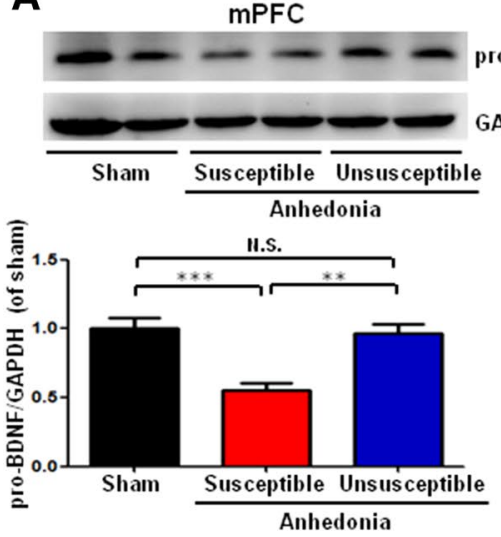

D

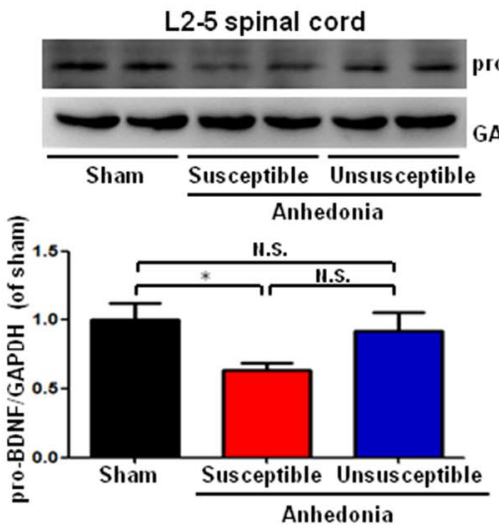

B
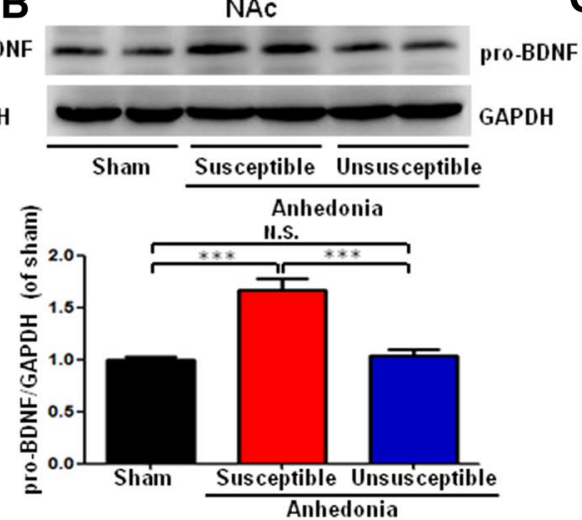

E

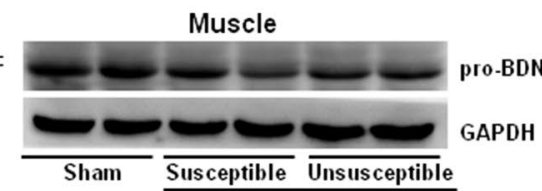

$\mathbf{F}$
C
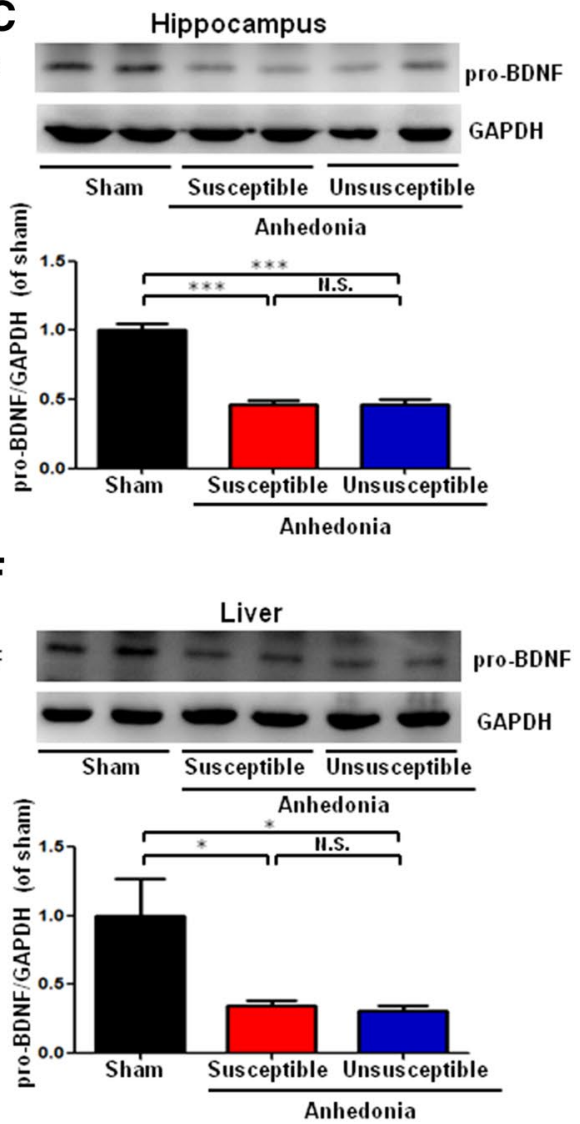

Fig. 3 Levels of proBDNF in selected tissues among the sham, anhedonia susceptible, and unsusceptible groups. a mPFC $\left(F_{(2,15)}=15.179, \quad P=0.001\right)$. b NAc $\quad\left(F_{(2,15)}=24.61, \quad P<0.001\right)$. c Hippocampus $\left(F_{(2,15)}=59.837, P<0.001\right)$. d L2-5 spinal cord $\left(F_{(2,15)}=3.021, P=0.079\right)$. e Muscle $\left(F_{(2,15)}=2.466, P=0.119\right)$. f
Liver $\left(F_{(2,15)}=6.11, P=0.031\right)$. Data are shown as mean \pm S.E.M. $(n=6) . * P<0.05, * * P<0.01$ or $* * * P<0.01$. proBDNF precursor of BDNF, $m P F C$ medial prefrontal cortex, NAc nucleus accumbens, N.S. not significant 
A
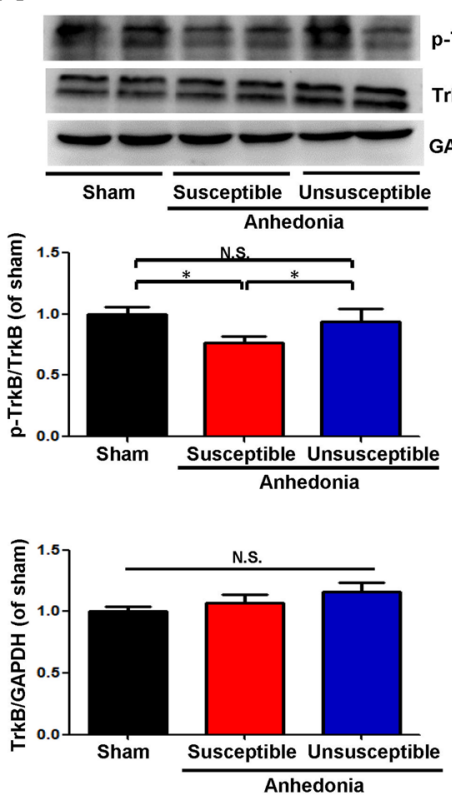

D
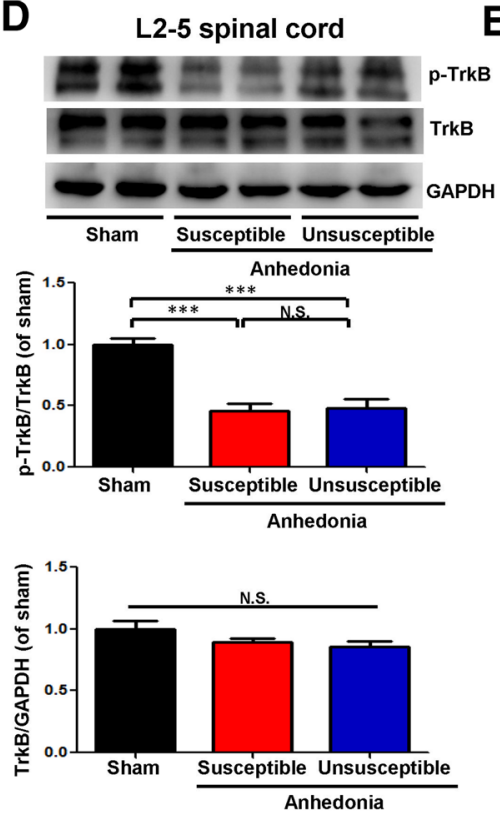

B
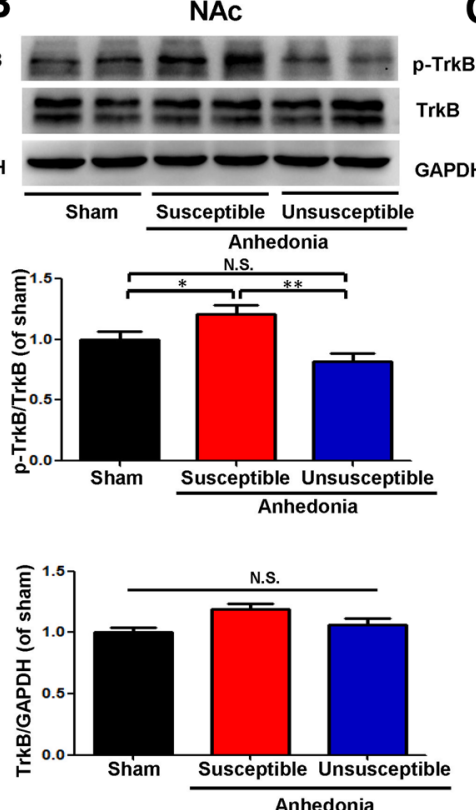

E
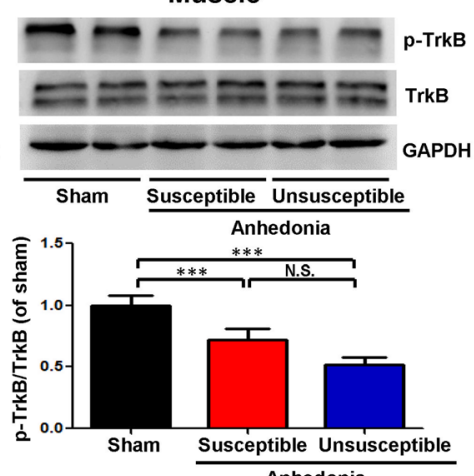

Anhedonia

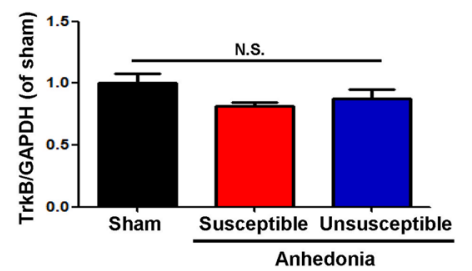

C
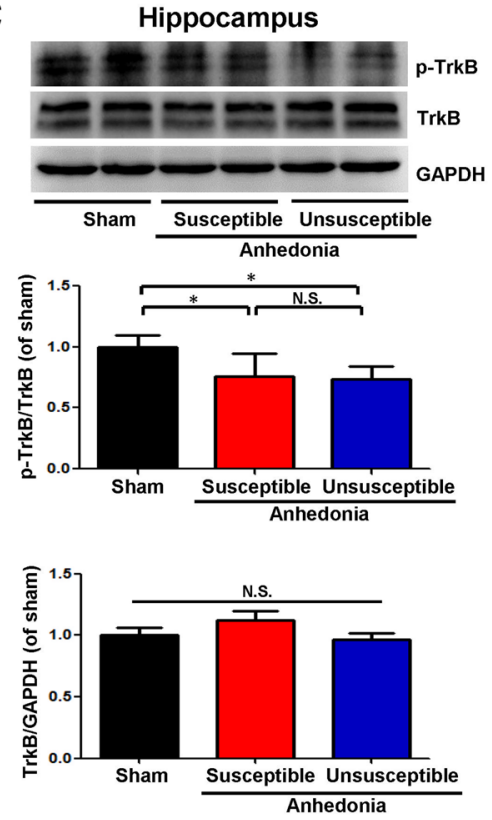

$\mathbf{F}$
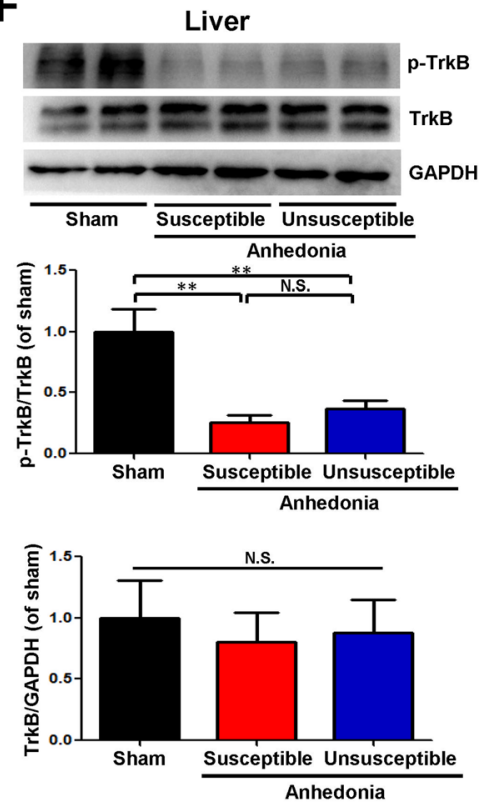

Fig. 4 Levels of p-TrkB and TrkB in selected tissues among the sham, anhedonia susceptible, and unsusceptible groups. a p-TrkB/ TrkB ratio $\left(F_{(2,15)}=6.994, P=0.027\right)$ and $\operatorname{TrkB}\left(F_{(2,15)}=1.565\right.$, $P=0.241)$ levels in the mPFC. b p-TrkB/TrkB ratio $\left(F_{(2,15)}=10.551\right.$, $P=0.002)$ and $\operatorname{TrkB}\left(F_{2,15}=1.729, P=0.211\right)$ levels in the NAc; c p-TrkB/TrkB ratio $\left(F_{(2,15)}=8.163, P=0.018\right)$ and $\operatorname{TrkB}\left(F_{(2,15)}=1.67\right.$, $P=0.221)$ levels in the hippocampus. d p-TrkB/TrkB ratio $\left(F_{(2,15)}=24.098, \quad P<0.001\right)$ and $\operatorname{TrkB}\left(F_{(2,15)}=2.376, \quad P=0.127\right)$

\section{Effects of 7,8-DHF and ANA-12 on the behavioral abnormalities in anhedonia susceptible rats after SNI}

Eighteen anhedonia susceptible rats were selected after levels in the L2-5 spinal cord. e p-TrkB/TrkB ratio $\left(F_{(2,15)}=9.747\right.$, $P=0.002)$ and TrkB $\left(F_{(2,15)}=2.133, P=0.153\right)$ levels in the muscle. f $\mathrm{p}$-TrkB/TrkB ratio $\left(F_{(2,15)}=11.089, P=0.001\right)$ and TrkB $\left(F_{(2,15)}=0.13, P=0.879\right)$ levels in the liver. Data are shown as mean \pm S.E.M. $(n=6)$. $* P<0.05, * * P<0.01$ or $* * * P<0.001 . m P F C$ medial prefrontal cortex, $N A c$ nucleus accumbens, N.S. not significant, $p$-TrkB phosphorylation-tropomyosin-receptor kinase B

hierarchical cluster analysis of SPT for further study (Fig. 5a, b). Anhedonia susceptible rats were treated with a single dose of 7,8-DHF (10 mg/kg) or ANA-12 $(0.5 \mathrm{mg} /$ $\mathrm{kg}$ ). 7,8-DHF significantly restored SNI-induced decrease in scores of MWT and SPT, while ANA-12 did not elicit 


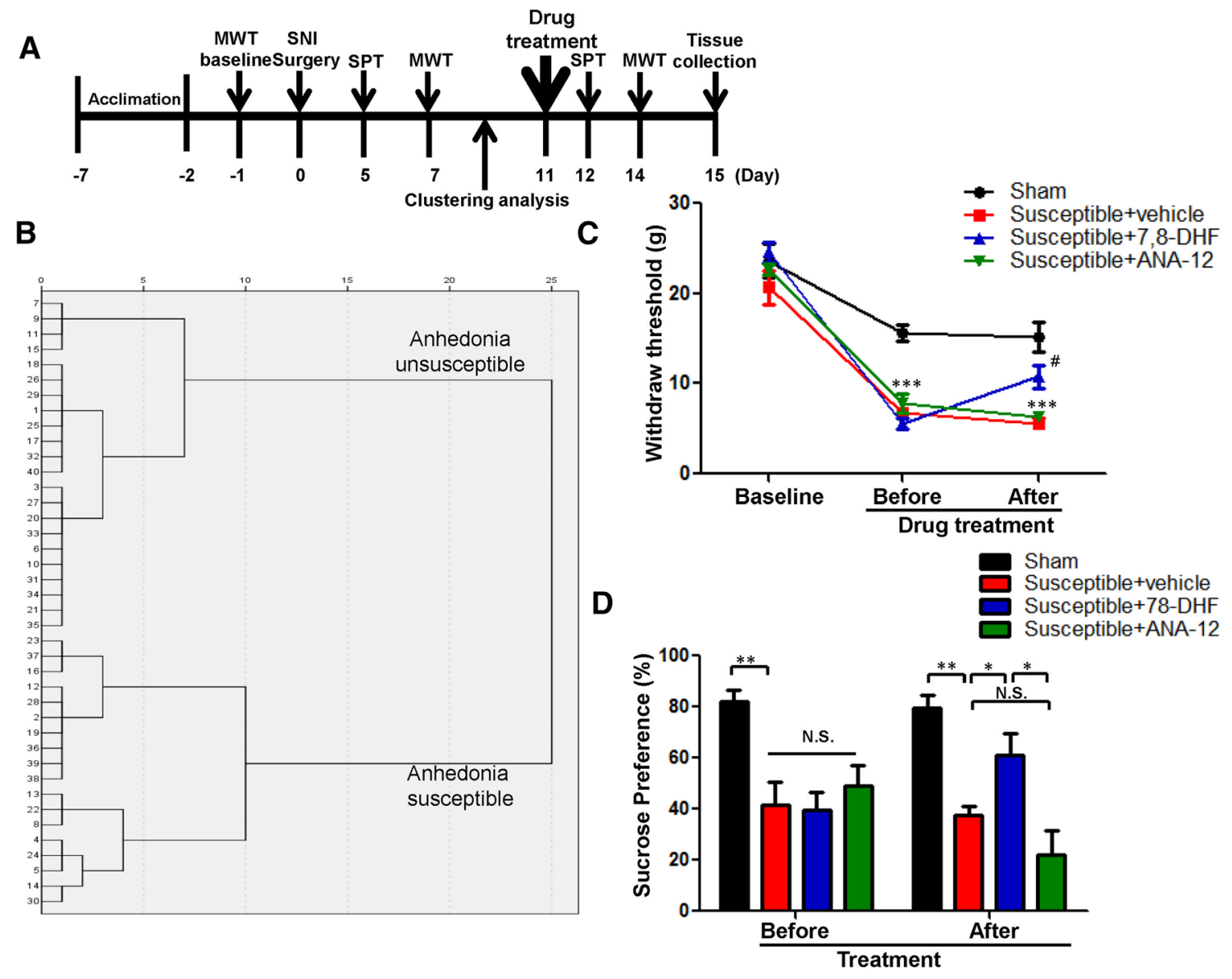

Fig. 5 Effects of 7,8-DHF and ANA-12 on behavioral abnormalities in anhedonia susceptible rats. a Schedule of SNI, MWT, and SPT. SNI was performed 7 day acclimation. MWT was measured on days 7 and 14 after SNI, respectively. SPT was performed on days 5 and 12 after SNI, respectively. Vehicle $(1 \mathrm{ml} / \mathrm{kg}), 7,8$-DHF $(10 \mathrm{mg} / \mathrm{kg})$, or ANA-12 $(0.5 \mathrm{mg} / \mathrm{kg})$ was intraperitoneally injected at a single dose on day 8. b Dendrogram of hierarchical clustering analysis. A total of 40 SNI rats were divided into anhedonia susceptible and unsusceptible groups by SPT results of hierarchical clustering analysis. c

MWT (time: $F_{(2,10)}=239.822, P<0.001$; Group: $F_{(3,15)}=20.158$, $P<0.001$; interaction: $\left.F_{(6,30)}=7.021, \quad P<0.001\right) . \quad * * * P<0.001$ v.s. sham group; ${ }^{\#} P<0.05$ v.s. susceptible + vehicle group. d SPT (time: $F_{(1,5)}=0.260, P=0.632$; Group: $F_{(3,15)}=11.040, P<0.001$; interaction: $\left.F_{(3,15)}=5.924, P=0.007\right) . * P<0.05, * * P<0.01$ or $* * * P<0.001$. Data are shown as mean \pm S.E.M. $(n=6)$. N.S. not significant, $S N I$ spared nerve injury, $S P T$ sucrose preference test, $M W T$ mechanical withdrawal test

these improving effects (Fig. 5c, d). The data suggest that 7,8-DHF, but not ANA-12, has anti-anhedonia effects in the anhedonia susceptible rats after SNI.

\section{Effects of 7,8-DHF and ANA-12 on the alterations in the BDNF-TrkB signaling in the MPFC and NAC of anhedonia susceptible rats}

To further elucidate the role of BDNF-TrkB signaling in the pathogenesis and therapeutic mechanisms of pain-induced anhedonia, we performed immunoblot analysis. Treatment with 7,8-DHF $(10 \mathrm{mg} / \mathrm{kg})$, but not ANA-12 $(0.5 \mathrm{mg} /$ $\mathrm{kg}$ ), significantly improved abnormal levels of BDNF and proBDNF, and the ratio of $\mathrm{p}-\mathrm{TrkB} / \mathrm{TrkB}$ in the mPFC of anhedonia-like phenotype rats after SNI (Fig. 6a-e). In

contrast, treatment of ANA-12, but not 7,8-DHF, significantly improved the abnormalities of BDNF-TrkB signaling in the NAc of anhedonia susceptible rats (Fig. 6f, j). However, there were no significant changes of total TrkB levels in the mPFC and NAc among the four groups (Fig. 6e, j). The data suggest that normalization of reduced BDNF-TrkB signaling in the mPFC by 7,8-DHF might play a role in its anti-anhedonia effects in the anhedonia susceptible rats.

\section{Discussion}

Although SNI rats suffered almost identical nociceptive damage, only some rats exhibited anhedonia-like phenotypes. Tissue levels of BDNF in the mPFC in rats with 


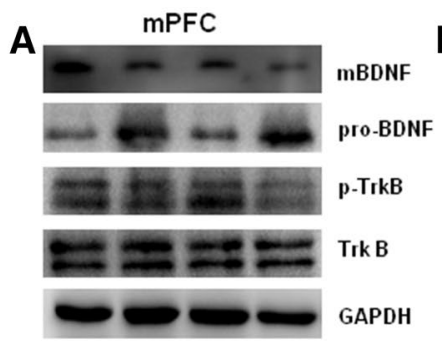

$\mathbf{F}$

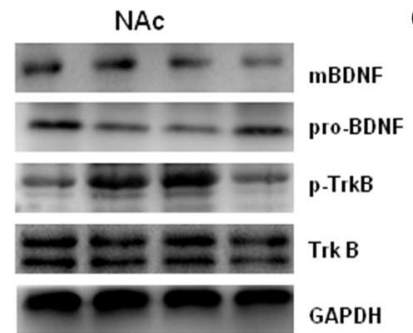

D
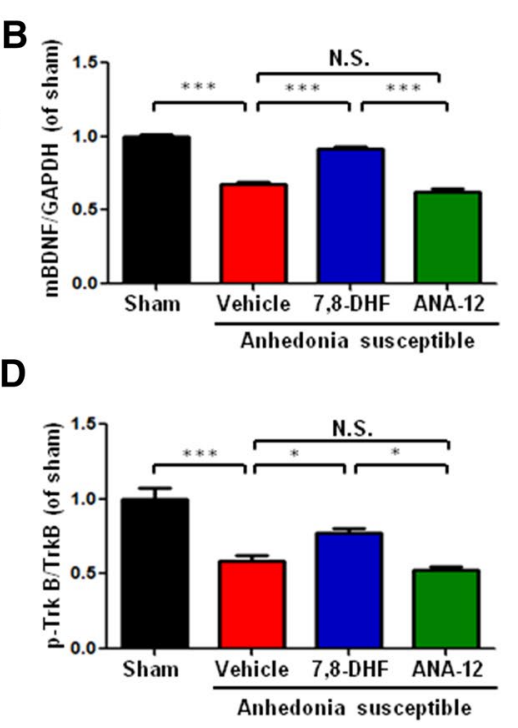

D

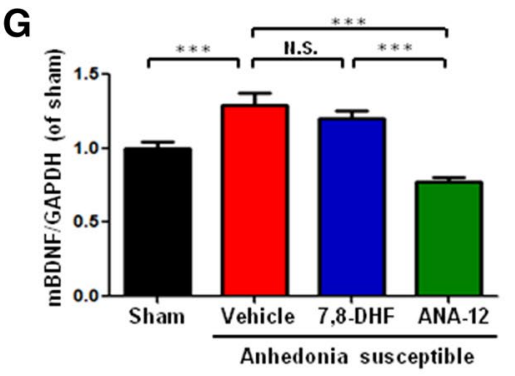

I

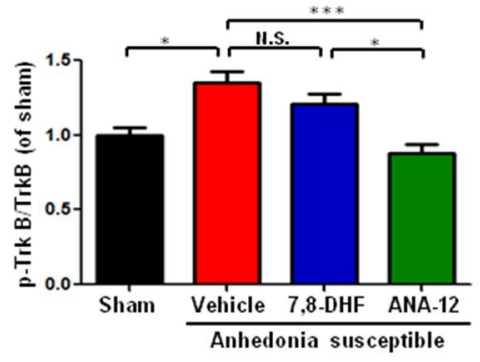

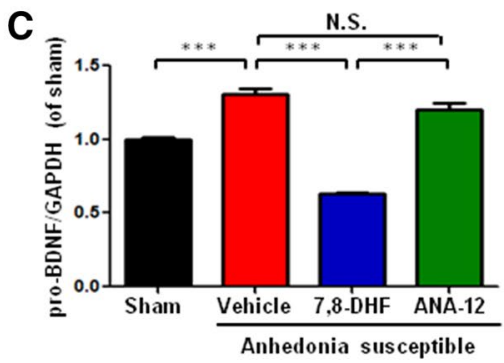

E

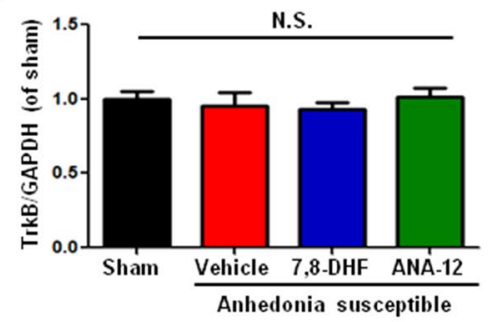

H

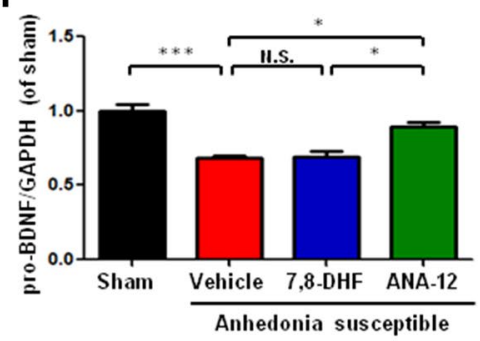

$J$

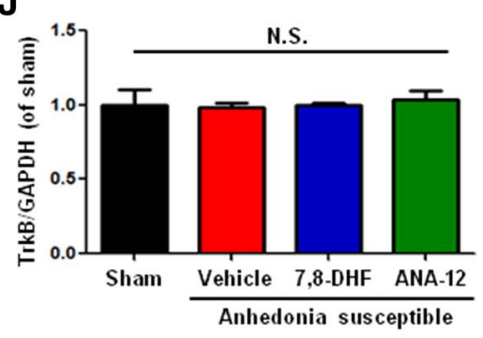

Fig. 6 Effects of 7,8-DHF and ANA-12 on BDNF-TrkB signaling in $\mathrm{mPFC}$ and NAc in anhedonia susceptible rats. a Western blot bands of BDNF-TrkB signaling in the mPFC. b BDNF level in the $\operatorname{mPFC}\left(F_{(3,20)}=119.273, P<0.001\right)$. c proBDNF level in the mPFC $\left(F_{(3,20)}=99.914, \quad P<0.001\right)$. d $\mathrm{p}-\operatorname{TrkB} / \operatorname{TrkB}$ ratio in the $\mathrm{mPFC}$ $\left(F_{(3,20)}=22.731, P<0.001\right)$. e TrkB level in the $\operatorname{mPFC}\left(F_{(3,20)}=0.340\right.$, $P=0.797)$. $\mathbf{f}$ Western blot bands of BDNF-TrkB signaling in the NAc. g BDNF level in the NAc $\left(F_{(3,20)}=17.414, P=0.001\right)$. h proBDNF

anhedonia-like phenotypes were lower compared with those in rats without anhedonia-like phenotypes and sham-operated rats. In contrast, tissue levels of BDNF in the NAc in rats with anhedonia-like phenotypes were higher compared with those in rats without anhedonia-like phenotypes and sham-operated rats. Furthermore, tissue levels of BDNF in the hippocampus, L2-5 spinal cord, muscle, and liver of rats with or without anhedonia-like phenotypes were lower compared with those of sham rats. Interestingly, a single level in the NAc $\left(F_{(3,20)}=21.974, P<0.001\right)$. i p-TrkB/TrkB ratio in the NAc $\left(F_{(3,20)}=10.828, P=0.003\right)$. $\mathbf{j}$ TrkB level in the NAc $\left(F_{(3,20)}=0.140, P=0.933\right)$. Data are shown as mean \pm S.E.M. $(n=6)$. $B D N F$ brain-derived neurotrophic factor, $m P F C$ medial prefrontal cortex, NAc nucleus accumbens, N.S. not significant, $p-T r k B$ phosphorylation-tropomyosin-receptor kinase $\mathrm{B}$, proBDNF precursor of BDNF; $* P<0.05$, ** $P<0.01$ or $* * * P<0.001$

dose of the TrkB agonist 7,8-DHF (10 mg/kg) ameliorated anhedonia-like behaviors and decreased BDNF-TrkB signaling in the mPFC of the rats with anhedonia-like phenotypes after SNI surgery. To the best of our knowledge, this is the first study showing the role of BDNF-TrkB signaling in individual differences of anhedonia after neuropathic pain. This is also the first study demonstrating the role of BDNF-TrkB signaling in the beneficial effects of 7,8-DHF in the comorbidity of neuropathic pain and depression in rodents. 
In preclinical studies, rats with hyperalgesia were highly heterogeneous in depression-related behaviors. Keay and colleagues reported that chronic constriction injury (CCI) induced a subgroup (approximately 30\%) of rats that had altered dominant behavior [8] and sleep-wake cycles [38] using resident-intruder social interaction and sleep-wake analyses. In this study, the hierarchical cluster analysis divided SNI rats into two clusters: one group (approximately $40 \%$, 'anhedonia-like phenotype') with reduced sucrose preference in the SPT, the other group (approximately $60 \%$, 'without anhedonia-like phenotype') with similar sucrose preference compared with sham-operated rats. The previous clinical studies demonstrated that the incidence of comorbid chronic pain and depression is around $30-50 \%[1,4$, 5], which is in line with the present study. In this study, we found that rats with or without anhedonia-like phenotypes showed similar mechanical withdrawal thresholds, suggesting that the alterations in mood-related behaviors were independent of the degree of nociceptive damage, consistent with the previous studies [8, 31, 38-40].

We found decreased BDNF-TrkB signaling in the mPFC and hippocampus from rats with anhedonia-like phenotypes on day 23 after SNI, consistent with the previous study [31]. Low levels of BDNF in the MPFC are associated with the development of depression-like phenotypes including anhedonia in rodents [19-28, 41]. In contrast, we found increased BDNF-TrkB signaling in the NAc from rats with anhedonialike phenotypes, consistent with the previous reports from rodents with depression-like phenotypes [19-28]. Interestingly, ANA-12 did not improve the decreased sucrose preference of rats with anhedonia-like phenotypes, although ANA12 significantly attenuated increased BDNF-TrkB signaling in the NAc from rats with anhedonia-like phenotypes. Thus, it is unlikely that BDNF-TrkB signaling in the NAc plays a role in the anhedonia-like phenotype after SNI. Taken together, it is likely that decreased BDNF-TrkB signaling in the mPFC might be implicated in the anhedonia-like phenotype in rats with neuropathic pain, and that TrkB agonists are potential therapeutic drugs for anhedonia in patients with neuropathic pain.

In this study, we found that both SNI rats with or without anhedonia-like phenotypes have lower tissue levels of BDNF in the L2-5 spinal cord compared to sham-operated rats. Given the role of BDNF-TrkB signaling in pain, it seems that reduced BDNF-TrkB signaling in the L2-5 spinal cord may play a role in the neuropathic pain, although BDNF-TrkB signaling in this region may not play a role in the anhedonialike phenotype.

As well as the brain, expression of proBDNF, BDNF, and its receptor TrkB has been reported in the liver and muscle [42-45], although the functional role of proBDNF-BDNFTrkB signaling in these tissues is not fully understood. The high expression of BDNF and TrkB proteins in the livers suggests neurotrophic support for autonomic innervation of this organ [43, 44]. A recent study suggests the role of BDNF-TrkB signaling in the synapse maintenance and function in the neuromuscular system [46]. Nonetheless, further detailed studies of the underlying role of proBDNF-BDNFTrkB signaling in these organs are needed. In addition, we also found both SNI rats with or without anhedonia-like phenotypes have lower tissue levels of BDNF in the muscle and liver compared with those in sham-operated rats. Interestingly, we observed alterations in BDNF-TrkB signaling in the liver from mood disorders including major depressive disorder and bipolar disorder, suggesting a possible role of BDNF-TrkB signaling in mood disorders [47]. Given the key role of BDNF-TrkB signaling in psychiatric disorders [48, 49], it is likely that alterations in BDNF-TrkB signaling may contribute to anhedonia susceptibility to SNI surgery. Further detailed studies on the role of BDNF-TrkB signaling in the brain as well as peripheral organs in the SNI model are needed.

In conclusion, the current study suggests that decreased BDNF-TrkB signaling in the mPFC plays key roles in individual differences of the anhedonia-like phenotype in rats with neuropathic pain. Therefore, it is likely that activation at BDNF-TrkB signaling could be a potential therapeutic target for anhedonia in patients with neuropathic pain.

Acknowledgements This study was supported by Grants from the National Natural Science Foundation of China [No.: 81571047 and 81771159 (to A.L.), 81500931 (to J.Z.), and 81703482 (to C.Y.)], and the Strategic Research Program for Brain Sciences from Japan Agency for Medical Research and Development, AMED (to K.H., JP17dm0107119).

\section{Compliance with ethical standards}

Conflict of interest Dr. Chun Yang received the research support from B. Braun Medical Inc. Other authors declared no conflict of interest.

Open Access This article is distributed under the terms of the Creative Commons Attribution 4.0 International License (http://creativeco mmons.org/licenses/by/4.0/), which permits unrestricted use, distribution, and reproduction in any medium, provided you give appropriate credit to the original author(s) and the source, provide a link to the Creative Commons license, and indicate if changes were made.

\section{References}

1. Bair MJ, Robinson RL, Katon W, Kroenke K (2003) Depression and pain comorbidity: a literature review. Arch Intern Med 163:2433-2445

2. Walker AK, Kavelaars A, Heijnen CJ, Dantzer R (2014) Neuroinflammation and comorbidity of pain and depression. Pharmacol Rev 66:80-101

3. Li JX (2015) Pain and depression comorbidity: a preclinical perspective. Behav Brain Res 276:92-98 
4. Radat F, Margot-Duclot A, Attal N (2013) Psychiatric co-morbidities in patients with chronic peripheral neuropathic pain: a multicentre cohort study. Eur J Pain 17:1547-1557

5. Gustorff B, Dorner T, Likar R, Grisold W, Lawrence K, Schwarz F et al (2008) Prevalence of self-reported neuropathic pain and impact on quality of life: a prospective representative survey. Acta Anaesthesiol Scand 52:132-136

6. Kroenke K, Wu J, Bair MJ, Krebs EE, Damush TM, Tu W (2011) Reciprocal relationship between pain and depression: a 12-month longitudinal analysis in primary care. J Pain 12:964-973

7. Keay KA, Monassi CR, Levison DB, Bandler R (2004) Peripheral nerve injury evokes disabilities and sensory dysfunction in a subpopulation of rats: a closer model to human chronic neuropathic pain? Neurosci Lett 361:188-191

8. Monassi CR, Bandler R. Keay KA (2003) A subpopulation of rats show social and sleep-waking changes typical of chronic neuropathic pain following peripheral nerve injury. Eur J Neurosci 17:1907-1920

9. Luedtke K, Bouchard SM, Woller SA, Funk MK, Aceves M, Hook MA (2014) Assessment of depression in a rodent model of spinal cord injury. J Neurotrauma 31:1107-1121

10. Nestler EJ, Barrot M, DiLeone RJ, Eisch AJ, Gold SJ, Monteggia LM (2002) Neurobiology of depression. Neuron 34:13-25

11. Hashimoto K, Shimizu E, Iyo M (2004) Critical role of brainderived neurotrophic factor in mood disorders. Brain Res Brain Res Rev 45:104-114

12. Duman RS, Monteggia LM (2006) A neurotrophic model for stress-related mood disorders. Biol Psychiatry 59:1116-1127

13. Martinowich K, Manji H, Lu B (2007) New insights into BDNF function in depression and anxiety. Nat Neurosci 10:1089-1093

14. Hashimoto K (2010) Brain-derived neurotrophic factor as a biomarker for mood disorders: an historical overview and future directions. Psychiatry Clin Neurosci 64:341-357

15. Hashimoto K (2013) Sigma-1 receptor chaperone and brainderived neurotrophic factor: emerging links between cardiovascular disease and depression. Prog Neurobiol 100:15-29

16. Castrén E (2014) Neurotrophins and psychiatric disorders. Handb Exp Pharmacol 220:461-479

17. Björkholm C, Monteggia LM (2016) BDNF-a key transducer of antidepressant effects. Neuropharmacology 102:72-79

18. Zhang JC, Yao W, Hashimoto K (2016) Brain-derived neurotrophic factor (BDNF)-TrkB signaling in inflammation-related depression and potential therapeutic targets. Curr Neuropharmacol 14:721-731

19. Liu WX, Wang J, Xie ZM, Xu N, Zhang GF, Jia M et al (2016) Regulation of glutamate transporter 1 via BDNF-TrkB signaling plays a role in the anti-apoptotic and antidepressant effects of ketamine in chronic unpredictable stress model of depression. Psychopharmacology 233:405-415

20. Zhang JC, Wu J, Fujita Y, Yao W, Ren Q, Yang Q et al (2014) Antidepressant effects of TrkB ligands on depression-like behavior and dendritic changes in mice after inflammation. Int J Neuropsychopharmacol 18:pyu077

21. Zhang JC, Yao W, Dong C, Yang C, Ren Q, Ma M et al (2017) Prophylactic effects of sulforaphane on depression-like behavior and dendritic changes in mice after inflammation. J Nutr Biochem 39:134-144

22. Ma M, Ren Q, Yang C, Zhang JC, Yao W, Dong C et al (2017) Antidepressant effects of combination of brexpiprazole and fluoxetine on depression-like behavior and dendritic changes in mice after inflammation. Psychopharmacology 234:525-533

23. Yang C, Shirayama Y, Zhang JC, Ren Q, Yao W, Ma M et al (2015) R-ketamine: a rapid-onset and sustained antidepressant without psychotomimetic side effects. Transl Psychiatry 5:e632

24. Ma M, Ren Q, Yang C, Zhang JC, Yao W, Dong C et al (2016) Adjunctive treatment of brexpiprazole with fluoxetine shows a rapid antidepressant effect in social defeat stress model: role of BDNF-TrkB signaling. Sci Rep 6:39209

25. Dong C, Zhang JC, Yao W, Ren Q, Ma M, Yang C et al (2017) Rapid and sustained antidepressant action of the mGlu2/3 receptor antagonist MGS0039 in the social defeat stress model: comparison with ketamine. Int $\mathbf{J}$ Neuropsychopharmacol $20: 228-236$

26. Shirayama Y, Yang C, Zhang JC, Ren Q, Yao W, Hashimoto K (2015) Alterations in brain-derived neurotrophic factor (BDNF) and its precursor proBDNF in the brain regions of a learned helplessness rat model and antidepressant effects of TrkB agonist and antagonist. Eur Neuropsychopharmacol 25:2449-2458

27. Yang C, Shirayama Y, Zhang JC, Ren Q, Hashimoto K (2015) Regional differences in brain-derived neurotrophic factor levels and dendritic spine density confer resilience to inescapable stress. Int J Neuropsychopharmacol 18:pyu121

28. Yang B, Yang C, Ren Q, Zhang JC, Chen QX, Shirayama Y et al (2016) Regional differences in the expression of brain-derived neurotrophic factor (BDNF) pro-peptide, proBDNF and preproBDNF in the brain confer stress resilience. Eur Arch Psychiatry Clin Neurosci 266:765-769

29. Merighi A, Salio C, Ghirri A, Lossi L, Ferrini F, Betelli C et al (2008) BDNF as a pain modulator. Prog Neurobiol 85:297-317

30. Trang T, Beggs S, Salter MW (2011) Brain-derived neurotrophic factor from microglia: a molecular substrate for neuropathic pain. Neuron Glia Biol 7:99-108

31. Xie ZM, Wang XM, Xu N, Wang J, Pan W, Tang XH et al (2017) Alterations in the inflammatory cytokines and brain-derived neurotrophic factor contribute to depression-like phenotype after spared nerve injury: improvement by ketamine. Sci Rep 7:3124

32. Spijker J, Bijl RV, De Graaf R, Nolen WA (2001) Determinants of poor 1-year outcome of DSM-III-R major depression in the general population: results of The Netherlands mental health survey and incidence study (NEMESIS). Acta Psychiatr Scand 103:122-130

33. McMakin DL, Olino TM, Porta G, Dietz LJ, Emslie G, Clarke $\mathrm{G}$ et al (2012) Anhedonia predicts poorer recovery among youth with selective serotonin reuptake inhibitor treatment-resistant depression. J Am Acad Child Adolesc Psychiatry 51:404-411

34. Jang SW, Liu X, Yepes M, Stepherd KR, Miller GW, Liu Y et al (2010) A selective TrkB agonist with potent neurotrophic activities by 7,8-dihydroxyflavone. Proc Natl Acad Sci USA 107:2687-2692

35. Cazorla M, Prémont J, Mann A, Girard N, Kellendonk C, Rognan D (2011) Identification of a low-molecular weight TrkB antagonist with anxiolytic and antidepressant activity in mice. J Clin Invest 121:1846-1857

36. Ren Q, Ma M, Yang C, Zhang JC, Yao W, Hashimoto K (2015) BDNF-TrkB signaling in the nucleus accumbens shell of mice has key role in methamphetamine withdrawal symptoms. Transl Psychiatry 5:e666

37. Fang X, Zhan G, Zhang J, Xu H, Zhu B, Hu Y et al (2018) Abnormalities in inflammatory cytokines confer susceptible to chronic neuropathic pain-related anhedonia in a rat model of spared nerve injury. Clin Psychopharmacol Neurosci (in press)

38. Austin PJ, Berglund AM, Siu S, Fiore NT, Gerke-Duncan MB, Ollerenshaw SL et al (2015) Evidence for a distinct neuro-immune signature in rats that develop behavioural disability after nerve injury. J Neuroinflamm 12:96

39. Austin PJ, Beyer K, Bembrick AL, Keay KA (2010) Peripheral nerve injury differentially regulates dopaminergic pathways in the nucleus accumbens of rats with either 'pain alone' or 'pain and disability'. Neuroscience 171:329-343

40. Gui WS, Wei X, Mai CL, Murugan M, Wu LJ, Xin WJ et al (2016) Interleukin- $1 \beta$ overproduction is a common cause for neuropathic 
pain, memory deficit, and depression following peripheral nerve injury in rodents. Mol Pain 12:12

41. Sun HL, Zhou ZQ, Zhang GF, Yang C, Wang XM, Shen JC et al (2016) Role of hippocampal p11 in the sustained antidepressant effect of ketamine in the chronic unpredictable mild stress model. Transl Psychiatry 6:e741

42. Yamamoto M. Sobue G, Yamamoto K, Terao S, Mitsuma T (1996) Expression of mRNAs for neurotrophic factors (NGF, BDNF, $\mathrm{NT}-3$, and GDNF) and their receptors (p75 ${ }^{\mathrm{NGFR}}$, trkA, trkB, and trkC) in the adult human peripheral nervous system and nonneural tissues. Neurochem Res 21:929-938

43. Cassiman D, Denef C, Desmer VJ, Roskams T (2001) Human and rat hepatic stellate cells express neurotrophins and neurotrophin receptors. Hepatology 33:148-158

44. Yang ZF, Ho DW, Lam CT, Luk JM, Lum CT, Yu WC et al (2005) Identification of brain-derived neurotrophic factor as a novel functional protein in hepatocellular carcinoma. Cancer Res 65:219-225
45. Genzer Y, Dadon M, Burg C, Chapnik N, O (2016) Effect of dietary fat and the circadian clock on the expression of brain-derived neurotrophic factor (BDNF). Mol Cell Endocrinol 430:49-55

46. Hurtado E, Cilleros V, Nadal L, Simó A, Obis T, Garcia N et al (2017) Muscle contraction regulates BDNF/TrkB signaling to modulate synaptic function through presynaptic $\mathrm{cPKC} \alpha$ and cPKC $\beta$ I. Front Mol Neurosci 10:147

47. Yang B, Ren Q, Zhang JC, Chen QX, Hashimoto K (2017) Altered expression of BDNF, BDNF pro-peptide and their precursor proBDNF in brain and liver tissues from psychiatric disorders: rethinking the brain-liver axis. Transl Psychiatry 7:e1128

48. Nijs J, Meeus M, Versijpt J, Moens M, Bos I, Knaepen K et al (2015) Brain-derived neurotrophic factor as a driving force behind neuroplasticity in neuropathic and central sensitization pain: a new therapeutic target? Expert Opin Ther Targets 19:565-576

49. Zhao H, Alam A, San CY, Eguchi S, Chen Q, Lian Q et al (2017) Molecular mechanisms of brain-derived neurotrophic factor in neuro-protection: Recent developments. Brain Res 1665:1-21 